\title{
THE RELATIONSHIP BETWEEN GAS AND CIRRUS DUST FILAMENTS AT THE EDGE OF THE EXPANDING SHELL IN ERIDANUS
}

\author{
G.L. Verschuur \\ Unaffiliated, USA \\ F. Verter \\ Laboratory for Astronomy \\ Goddard Space Flight Center, USA
}

\author{
L.J. Rickard \\ Naval Research Laboratory, USA \\ D.T. Leisawitz \\ Laboratory for Astronomy \\ Goddard Space Flight Center, USA
}

\begin{abstract}
At the boundary of a large expanding shell in Eridanus around $l=187^{\circ}, b=-50^{\circ}$ the morphology observed in the $\mathrm{H}$ I emission is well mimicked by the $100 \mu \mathrm{m}$ surface brightness but with associated structures offset by as much as $0 .{ }^{\circ} 5$. A point-to-point comparison between $I_{100 \mu \mathrm{m}}$ and $N_{\mathrm{HI}}$ in filaments of neutral hydrogen and dust (IR cirrus) produces only a weak dependence. However, when $I_{100 \mu \mathrm{m}}$ at a cirrus dust peak is compared with $N_{\mathrm{HI}}$ at the associated $\mathrm{H}$ I peak, a relationship closer to that reported by other workers is found. Preliminary $\mathrm{CO}$ observations have set low limits on the molecular gas in these filaments. Since the H I and dust in our region are associated with a large expanding shell (or superbubble), shocks may be responsible for separation of gas and dust. The existence of small-scale structure in both the H I and IR is noted. We conclude that attempts to correlate H I and IR must invoke high-resolution area surveys.
\end{abstract}

\section{OBSERVATIONS}

Fully mapped, narrow velocity, H I filaments in an area of about $140 \mathrm{deg}^{2}$ around $l=187^{\circ}$, $b=-50^{\circ}$ (Verschuur 1973) are known to be associated with $\mathrm{H} \alpha$ filaments. We have examined the $100 \mu \mathrm{m}$ emission obtained from the Infrared Astronomical Satellite (IRAS) in the same region and find that each segment of the $\mathrm{H}$ I filaments has an equivalent IR structure of the same general length and width but sometimes offset in position by up to $0 .^{\circ} 5$. The typical angular width of the $\mathrm{HI}$ and IR filaments ranges from 0.5 to $1^{\circ} .2$. These two components are, in turn, offset by as much as $3 .^{\circ} 5$ from parallel $\mathrm{H} \alpha$ filaments. The overall parallelism between $\mathrm{H} \alpha$ and IR emission is maintained over the $50^{\circ}$ angular extent of the major filament defining the ridge of Eridanus as was determined by comparing the $\mathrm{H} \alpha$ data of Reynolds and Ogden (1979) with IRAS data.

Comparison of the detailed morphology of the $\mathrm{H} \mathrm{I}$ and IR structures within the limited area we have studied shows that virtually every peak and valley in the $\mathrm{H} \mathrm{I}$ distribution can be identified in the IRAS data but with offsets that vary from 0 to $\pm 0.0^{\circ} 5$ across the region.

A point-to-point comparison between $I_{100 \mu \mathrm{m}}$ and $N_{\mathrm{HI}}$ in the Eridanus filaments gives

$$
I_{100 \mu \mathrm{m}}=0.15( \pm 0.06) N_{\mathrm{HI}} \times 10^{20} \mathrm{~cm}^{-2} / \mathrm{MJy} \mathrm{sr}^{-1} \text {, }
$$

where the error is two standard deviations. The coefficient of 0.15 compares with values of 1.0 found by Deul (1988) and 1.07 by de Vries, Heithausen, and Thaddeus (1987). We also identified 28 pairs of peaks and 11 pairs of minima in the two maps and measured their positions. The relationship between $I_{100 \mu \mathrm{m}}$ and $N_{\mathrm{HI}}$ in offset but associated peaks is described by

$$
I_{100 \mu \mathrm{m}}=0.55( \pm 0.10) N_{\mathrm{HI}} \times 10^{-20} \mathrm{~cm}^{-2} / \mathrm{MJy} \mathrm{sr}^{-1} \text {. }
$$

The coefficient is now closer to that found by others.

Preliminary CO observations show that these filaments contain no significant amounts of $\mathrm{H}_{2}$ that would help account for the apparent separation between $\mathrm{H}$ I and dust. 


\section{COMPARISON WITH OTHER WORK}

Apparent separation between H I and IR filaments has also been found by others, for example, by de Geus (1988) in the Scorpio-Centaurus region, where a major filament of $\mathrm{H} \mathrm{I}$ is offset from a CO and IR counterpart by $2^{\circ}$ over a $12^{\circ}$ length. The distance of this material was estimated to be 80 and $125 \mathrm{pc}$, which compares with $400 \mathrm{pc}$ for the Eridanus region (Reynolds and Ogden 1979), although we cannot rule out that the Eridanus filaments are as close as 150 pc. The physical offsets are therefore of the same order in both regions, each of which is associated with a center of star formation and exhibiting shell-like patterns indicative of shock activity. The anti-coincidence between $\mathrm{H}$ I and IR emission is even more dramatically evident in the data of de Vries, Heithausen, and Thaddeus (1987) in the clouds in Ursa Major. Although several IR features appear to be associated with $\mathrm{H}$ I structures, others clearly are not.

\section{DISCUSSION}

Pending a full theoretical analysis, we suggest that the possible separation between the $\mathrm{H}$ I and the cirrus in Eridanus can be explained by the action of shocks. The greater mass of the grain insures that it experiences less deflection per collision; consequently, the gas is swept up by the shock whereas the bigger grains tend to be left behind. This model implies a post-shock offset of the gas from the dust, with the dust closer to the origin of the shock. The filamentary structures are long and narrow coherent entities, elongated parallel to the magnetic field direction (as inferred from optical polarization vectors); they retain a remarkably uniform velocity (within a few $\mathrm{km} \mathrm{s}^{-1}$ ) over their length and may be due to shocks at the edge of the expanding Eridanus shell. Without being shock-driven, it is difficult to imagine that they would retain their coherence either over the physical lengths involved or over long time scales.

\section{CONCLUSIONS}

Our high-resolution data suggest considerable structure in the $\mathrm{H} \mathrm{I}$ and $100 \mu \mathrm{m}$ emission brightness in the Eridanus filaments. When the IR emission is compared with the $\mathrm{H}$ I there is little direct positional agreement. At first glance it appears that the brightest $100 \mu \mathrm{m}$ emission lies along the edges of the neutral hydrogen filaments. We are not yet convinced that we can account for apparent variations in IR brightness as resulting exclusively from temperature effects, in part because of the relationship between the filaments and because structure within them is so complex.

If gas-dust offsets seen here and elsewhere are real, great caution must be exercised in interpreting low resolution studies of the relationship between $\mathrm{H}$ I and IR emission. High resolution $21 \mathrm{~cm}$ survey data are essential if we are to draw conclusions relating to gas-to-dust ratios in interstellar space.

\section{REFERENCES}

Deul, E. 1988, Ph. D. dissertation, University of Leiden.

de Geus, E. 1988, Ph. D. dissertation, University of Leiden.

Reynolds, R. J. and Ogden, P. M. 1979, Ap. J., 229, 942.

Verschuur, G. L. 1973, Astron. J., 78, 573. (A +4 minute correction must be applied to the right ascension scale of the $\mathrm{H}$ I contour maps in this paper.)

de Vries, H. W., Heithausen, A., and Thaddeus, P. 1987, Ap. J., 319, 723. 